AperTO - Archivio Istituzionale Open Access dell'Università di Torino

\title{
p130Cas promotes invasiveness of three dimensional ErbB2-transformed mammary acinar structures by enhanced activation of mTOR/p70S6K and Rac1
}

\section{This is the author's manuscript}

Original Citation:

Availability:

This version is available http://hdl.handle.net/2318/85004

since

Published version:

DOI:10.1016/j.ejcb.2010.09.002

Terms of use:

Open Access

Anyone can freely access the full text of works made available as "Open Access". Works made available under a Creative Commons license can be used according to the terms and conditions of said license. Use of all other works requires consent of the right holder (author or publisher) if not exempted from copyright protection by the applicable law. 


\section{(30) \\ UNIVERSITÀ DEGLI STUDI DI TORINO}

This Accepted Author Manuscript (AAM) is copyrighted and published by Elsevier. It is posted here by agreement between Elsevier and the University of Turin. Changes resulting from the publishing process - such as editing, corrections, structural formatting, and other quality control mechanisms - may not be reflected in this version of the text. The definitive version of the text was subsequently published in [Eur J Cell Biol. 2011 Feb-Mar;90(2-3):237-48. doi: 10.1016/j.ejcb.2010.09.002. p130Cas promotes invasiveness of three-dimensional ErbB2-transformed mammary acinar structures by enhanced activation of mTOR/p70S6K and Rac1.

$\underline{\text { Tornillo } G^{I}}, \underline{\text { Bisaro B }}, \underline{\text { Camacho-Leal Mdel P }}, \underline{\text { Galiè } M}$, Provero P, Di Stefano P, Turco E, Defilippi P, Cabodi .

You may download, copy and otherwise use the AAM for non-commercial purposes provided that your license is limited by the following restrictions:

(1) You may use this AAM for non-commercial purposes only under the terms of the CC-BY-NC-ND license.

(2) The integrity of the work and identification of the author, copyright owner, and publisher must be preserved in any copy.

(3) You must attribute this AAM in the following format: Creative Commons BY-NC-ND license (http://creativecommons.org/licenses/by-nc-nd/4.0/deed.en), [+ Digital Object Identifier link to the published journal article on Elsevier's ScienceDirect ${ }^{\circledR}$ platform $]$ 
p130Cas promotes invasiveness of three dimensional ErbB2-transformed mammary acinar structures by enhanced activation of mTOR/p70S6K and Rac1

Giusy Tornillo, Brigitte Bisaro, Maria del Pilar Camacho Leal, Mirco Galiè ${ }^{1}$, Paolo Provero, Paola Di Stefano, Emilia Turco, Paola Defilippi * \& Sara Cabodi *

Molecular Biology Center, Dept. of Genetics, Biology and Biochemistry, University of Torino, Via Nizza 52, Torino

1 Department of Clinical and Experimental Medicine, Section of Haematology, University of Verona, Italy

*co-corresponding authors 


\begin{abstract}
ErbB2 over-expression is detected in approximately $25 \%$ of invasive breast cancers and is strongly associated with poor patient survival. We have previously demonstrated that p130Cas adaptor is a crucial mediator of ErbB2 transformation. Here, we analyzed the molecular mechanisms through which p130Cas controls ErbB2-dependent invasion in three-dimensional cultures of mammary epithelial cells. Concomitant p130Cas over-expression and ErbB2 activation enhance PI3K/Akt and Erk1/2 MAPK signalling pathways and promote invasion of mammary acini. By using pharmacological inhibitors, we demonstrate that both signalling cascades are required for the invasive behaviour of p130Cas over-expressing and ErbB2 activated acini. Erk1/2 MAPK and PI3K/Akt signalling triggers invasion through distinct downstream effectors involving mTOR/p70S6K and Rac1 activation, respectively. Moreover, in silico analyses indicate that p130Cas expression in ErbB2 positive human breast cancers significantly correlates with higher risk to develop distant metastasis, thus underlying the value of the p130Cas/ErbB2 synergism in regulating breast cancer invasion. In conclusion, high levels of p130Cas favour progression of ErbB2 transformed cells towards an invasive phenotype.
\end{abstract}

Keywords: p130Cas, ErbB2, tumour invasion, breast cancer, three-dimensional culture 


\section{Introduction}

The ErbB2 oncogene (also known as HER2) belongs to the epidermal growth factor-receptor family and its over-expression and activation have been detected in a large subset of mammary cancers (Hynes and Lane, 2005). Amplification or over-expression of the ErbB2 gene has been detected in many cancers, including human breast, lung and gastric cancers and is associated with increased tumor grade and shorter overall survival rates (Allgayer et al., 2000; Sharma and Settleman, 2009; Slamon et al., 1989). ErbB2 activation is associated with an increase of its tyrosine phosphorylation and results in the phosphorylation of a host of downstream molecules, which in turn activate a variety of signalling cascades, including phosphatidylinositol-3 kinase (PI3K)/Akt, Src kinase, phospholipase $\mathrm{C}$ gamma and the RAS/mitogen activated protein kinase cascade (Baselga and Swain, 2009; Hynes and Lane, 2005; Hynes and MacDonald, 2009; Neve et al., 2002; Yarden and Sliwkowski, 2001).

p130Cas was originally identified as a tyrosine phosphorylated protein upon transformation by vand v-Crk oncogenes and for its ability to associate with Crk (Matsuda et al., 1990; Reynolds et al., 1989). p130Cas is a multifunctional adaptor protein required for embryonic development (Honda et al., 1998) and is characterized by structural motifs that enable interactions with a variety of signaling molecules. These multi-protein complexes sense and integrate signalling originating from several receptor systems (Bouton et al., 2001; Defilippi et al., 2006; O'Neill et al., 2000).

p130Cas functions as a molecular scaffold within focal adhesion complexes, and is readily phosphorylated by focal adhesion kinase (FAK) and c-Src (Mitra and Schlaepfer, 2006). For its inclusion in focal adhesion complexes, p130Cas has also been proposed to play a critical role in mediating cell migration and invasion. Indeed, fibroblasts derived from p130Cas-deficient embryos exhibit drastically altered cytoskeletal architectures (Honda et al., 1998) and after transformation with c-Src become significantly more invasive when engineered to simultaneously over-express p130Cas (Brabeck 2004). In addition, p130Cas association with Crk constitutes a molecular switch for cell motility by recruiting DOCK180 to integrin-containing adhesion complexes (Klemke et al., 
1998). Moreover, c-Src-mediated p130Cas phosphorylation and the assembly of a p130Cas-CrkDOCK180 scaffold drive cell migration and responses to mechanical stress (Mitra and Schlaepfer, 2006; Stupack et al., 2000)

Recent data support a role for p130Cas in the acquirement of resistance to breast cancer therapy. It has been shown that patients with primary breast tumors expressing high levels of p130Cas (also known as BCAR-1) experience a more rapid disease recurrence and have a greater risk of resistance to tamoxifen therapy (van der Flier et al., 2000). Moreover, over-expression of p130Cas/BCAR1 can confer breast cancer resistance to adriamycin (Ta et al., 2008).

We have previously shown that double transgenic mice originated by crossing MMTV-p130Cas and MMTV-NeuT mice, that express the oncogenic form of the rat neu gene, homologous to human ErbB2 (Muller et al., 1988; Nanni et al., 2000), showed an accelerated onset of mammary tumour formation (Cabodi et al., 2006). In addition, the analysis of human breast samples revealed that tumours over-expressing both p130Cas and ErbB2 are characterised by an elevated proliferation index (Cabodi et al., 2006). We have also recently shown that p130Cas is an essential transducer element in ErbB2 transformation, demonstrating that p130Cas is necessary for ErbB2-dependent foci formation, anchorage-independent growth, in vivo tumour growth and lung colonisation (Cabodi etal., 2010).

The human mammary cells MCF10A.B2 that express a chimeric form of ErbB2, which can homodimerize in the presence of the synthetic ligand AP1510 (Muthuswamy et al., 2001), are a suitable in vitro model for the study the ErbB2-dependent transformation in three-dimensional (3D) matrix instead of the classical monolayer culture. Here, we uncover the molecular mechanisms for p130Cas-dependent invasiveness of ErbB2 transformed cells showing that p130Cas drives invasion of ErbB2-transformed mammary acinar structures by enhancing activation of mTOR/p70S6K and Rac1. 


\section{Material and Methods}

\section{Antibodies and reagents}

Mouse monoclonal antibodies to p130Cas and beta-1 integrin and rabbit polyclonal antibodies to fibronectin were produced at the MBC, University of Torino. Phospho Src (Y416), phospho Erk1/2 (T202/T204), phospho Akt (S473), phospho S6Rp (S235/236), S6Rp, Erk1/2 MAPK, Akt, phospho p70S6K T389 and T421/S424, p70S6K antibodies were obtained from Cell Signaling (Beverly, MA), Rac1 antibody was from Millipore (Billerica, MA, USA). c-Src antibody was from Santa Cruz (Palo Alto, CA, USA). Rac inhibitor (NSC23766) was from Calbiochem (Merck KGaA, Darmstadt, Germany), MAPKK inhibitor (PD98059), Src inhibitor (SU6656) and PI3K inhibitor (LY294002) from Sigma (St. Louis, MO, USA), Rapamycin was from Cell signalling. Matrigel and collagen I were purchased from BD Trasduction Laboratories (Franklin Lakes, NY). Secondary antibodies conjugated with peroxidase were purchased from GE Healthcare. Alexa Fluor 633 phalloidin and Alexa Fluor Dye secondary antibodies were from Invitrogen (Carlsbad, CA, USA).

\section{Cell cultures}

MCF10A.B2 engineered cells were kindly provided by Dr. Muthuswamy (Cold Spring Harbor Laboratory), (Muthuswamy et al., 2001). MCF10A.B2 cells were maintained in DMEM/F12 (Gibco, BRL) supplemented with $5 \%$ horse serum, $20 \mathrm{ng} / \mathrm{ml}$ EGF, $10 \mu \mathrm{g} / \mathrm{ml}$ insulin, $1 \mathrm{ng} / \mathrm{ml}$ cholera toxin, $100 \mu \mathrm{g} / \mathrm{ml}$ hydrocortisone, $50 \mathrm{U} / \mathrm{ml}$ penicillin and $50 \mu \mathrm{g} / \mathrm{ml}$ streptomycin. To generate 10A.B2Mock and 10A.B2Cas cells, we transduced MCF10A.B2 with pBabe retroviral empty vector or carrying p130Cas cDNA fused with GFP.

\section{Three-dimensional morphogenetic assay}

3D morphogenetic assays were conducted as previously described in http://muthuswamylab.cshl.edu/ml_protocols.html and in (Debnath et al., 2003; Seton-Rogers et al., 2004). Phase images were collected by using Zeiss microscopy at $4 \mathrm{X}$ magnitude.

\section{Immunobloting analysis from 3D cultures}


3D acini were released from Matrigel:Collagen using BD cell recovery solution (BD Biosciences). Protein extracts were obtained by lysing acini with RIPA buffer $(50 \mathrm{mM}$ Tris-HCl $\mathrm{pH} 7.4,150 \mathrm{mM}$ $\mathrm{NaCl} ; 1 \%$ Triton $\mathrm{X}-100$, and protease inhibitors). Total cell lysates were separated by SDS-PAGE, transferred to nitrocellulose, and immunoblotted overnight with primary antibodies at $4^{\circ} \mathrm{C}$. Blots were incubated with mouse or rabbit horseradish-peroxidase conjugated secondary antibodies for 1 hour at room temperature. ECL (Euroclone) was used to detect chemoluminescent signals. Protein band intensities were determined using the Image $\mathbf{J}$ software developed by the NIH.

$\underline{\text { Immunofluorescence analysis of 3D acinar structures }}$

Immunostaining of 3D cultured MCF10.B2 cells was performed as described in http://muthus wamylab.cshl.edu/protocols/IF\% 20protocol.pdf . Images were taken at HCX PL APO CS 63X 1.4 OIL Leica TCS-SP5 II confocal microscope and analyzed with LASAF software.

\section{Rac pull-down assay}

Rac1 pull-down assay was performed as described in (Di Stefano et al., 2007). Briefly, glutathionecoupled Sepharose 4B beads bound to recombinant GST-PAK CRIB domain fusion proteins were incubated with cell extracts at $4^{\circ} \mathrm{C}$ for $45 \mathrm{~min}$, eluted in Laemmli buffer and analysed for the presence of Rac1 by Western blot.

\section{In silico analysis}

The NKI dataset for early stage breast cancer containing 295 breast cancer specimens, analysed on a 25,000 spot oligonucleotide array as described previously, was used (van de Vijver et al., 2002). Only the 102 patients positive for ErbB2 expression (expression above the mean) were considered. The difference in ErbB2 expression between patients with high levels of p130Cas and with low levels of p130Cas (with respect to the mean expression level across the samples) was determined with a two-sided Mann-Whitney U test. 
Distant metastasis-free survival times was defined as the time to the first event. Overall survival times were based on death from any cause. Patients were censored at last follow up. Kaplan-Meier survival curves were compared using a log-rank test (R survival package). 


\section{Results}

In human breast tumours p130Cas and ErbB2 co-expression correlates with metastasis and poor prognosis

Our previous data indicate that p130Cas is involved in ErbB2 tumorigenesis (Cabodi et al., 2006) and is required for lung colonisation of ErbB2-transformed cells (Cabodi et al., 2010). The clinical relevance of these findings is supported by the in silico analysis that we performed using publicly available microarray data from the Netherlands Cancer Institute of 295 early stage breast cancer biopsies (van de Vijver et al., 2002). On this set of data we evaluated the expression of p130Cas in patients that were positive for ErbB2 (102 out of 295). Interestingly, as shown in the boxplot in Figure 1A, the expression of ErbB2 is significantly higher in patients with high levels of p130Cas $\left(\mathrm{p} 130 \mathrm{Cas} / \mathrm{BCAR} 1^{+}\right)$compared to patients with low levels of p130Cas (p130Cas/BCAR1 ${ }^{-}$) $(\mathrm{P}=0.0010$ from Mann-Whitney $\mathrm{U}$ test) (Figure 1A). Moreover, as visualized with Kaplan-Meier plots, patients with breast cancers with concomitant elevated expression of p130Cas and ErbB2 have a shorter overall survival $(P=0.012$, Figure $1 \mathrm{~B})$ and, interestingly, a higher risk of developing distant site metastasis $(P=0.0445$, Figure 1C) compared to patients with low levels of p130Cas. Thus, ErbB2 and p130Cas co-expression in this cohort of breast cancer patients significantly correlates with metastasis and poor prognosis, supporting the relevance of p130Cas and ErbB2 cooperation in promoting breast cancer progression.

p130Cas over-expression and ErbB2 activation promote 10A.B2 acini invasion by enhancing Erk1/2 MAPK and PI3K/Akt signalling pathways

To investigate the mechanisms through which high levels of p130Cas and ErbB2 lead to cell invasion, we used the MCF10A.B2 grown in 3D (Muthuswamy et al., 2001). MCF-10A.B2 (10A.B2Mock) cells grown in a 3D matrix composed by Matrigel and collagen I (1:1) form polarized, quiescent acini-like spheroids, that upon activation of ErbB2 by treatment with the synthetic ligand AP1510, undergo re-initiation of proliferation and disruption of apical polarity, 
without invading the surrounding matrix (Muthuswamy et al., 2001). In contrast, 10A.B2Cas cells that express exogenous p130Cas fused to GFP give rise to multiacinar structures, that upon stimulation with AP1510 acquire invasive protrusions (Figure 2B). Migration and invasion of mammary epithelial cells transformed by ErbB2 have been ascribed to activation of several signalling pathways, including the Extracellular regulated kinase Erk1/2 MAPK and PI3K/Akt (Ignatoski et al., 2000; Spencer et al., 2000). Hence, we analysed the activation of these signalling pathways in our experimental model. 10A.B2Mock and 10A.B2Cas derived acini were treated either with vehicle or with AP1510 and protein extracts from 3D cultures were analysed for levels of p130Cas and activation of Akt and Erk1/2 MAPK by using specific antibodies. Interestingly, upon ErbB2 activation, phosphorylation of both Akt and Erk1/2 was markedly increased in 10A.B2Cas compared to 10A.B2Mock acini (Figure 2A). Notably, in this 3D model, p130Cas overexpression does not enhance the activity of other known ErbB2 induced signalling molecules, such as c-Src. c-Src is indeed activated in 10A.B2 cells by treatment with AP1510, but its activation is not further enhanced by the over-expression of p130Cas, at least at the time of our analysis $\underline{\text { Figure }}$ $\underline{\text { S1B)}}$. Further, to determine the relevance of the activation of PI3K/Akt and Erk1/2 MAPK signalling pathways in conferring the invasive properties to acini over-expressing p130Cas in response to ErbB2 activation, 10A.B2Cas acini stimulated with AP1510 were treated with 25 micromolar PD98059, a MEK-specific inhibitor, or with 10 micromolar LY294002, which blocks PI3K activity. As shown in Figure 2B, the treatment with either one inhibitor was sufficient to block the formation of invasive protrusions in AP1510-treated 10A.B2Cas cells, indicating that PI3K and Erk1/2 cascades are both involved in regulating the invasive behaviour of mammary epithelial cells upon concomitant p130Cas over-expression and ErbB2 activation.

\section{ErbB2-induced invasion of 10A.B2Cas acini requires p70S6K activity}

The mammalian target of rapamycin (mTOR) is a 289-kDa serine/threonine kinase that belongs to the phosphoinositide 3-kinase (PI3K)-related kinase family and is conserved 
throughout evolution (Ma et al., 2009; Laplante et al., 2009). mTOR signalling pathway integrates and responds to both extracellular and intracellular cues and serves as a central regulator of cell metabolism, growth, proliferation and survival. One of the main downstream target of mTOR is the p70S6 kinase (p70S6K), involved in the physiological control of protein synthesis. (Ma et al., 2009; Laplante et al., 2009).

Increasing evidences indicate that both the PI3K/Akt and the Erk1/2 MAPK signalling can activate mTOR/p70S6K pathway (Klos et al., 2006; Lehman and Gomez-Cambronero, 2002; Sekulic et al., 2000). Deregulation of the mTOR/p70S6K pathway may play an important role in cancer progression as well as in many other diseases (Hay and Sonenberg, 2004; Holland et al., 2004). Activation of $\mathrm{p} 70 \mathrm{~S} 6 \mathrm{~K}$ is a multistep process starting with the priming phosphorylation on residues T421 and S424 within the auto-inhibitory domain by kinases such as Erk1/2 MAPKs, followed by phosphorylation of T389 by mTOR that is critical for p70S6K activation (Pearson et al., 1995; Saitoh et al., 2002; Thomas, 1993). Although ErbB2 has been shown to activate mTOR/p70S6K (Klos et al., 2006), the role of mTOR/p70S6K in ErbB2-mediated cancer invasion remains unclear. Consequently, to analyze the effect of p130Cas over-expression on ErbB2-dependent activation of the p70S6K pathway, 3D cultures of 10A.B2Mock and 10A.B2Cas were treated or untreated with AP1510. p70S6K activity was assessed in cell extracts using phosphospecific antibodies directed against p70S6K and the ribosomal subunit protein S6 (S6Rp), a known downstream target of p70S6K. Our results indicate that upon ErbB2 activation, p70S6K and its substrate S6Rp are more phosphorylated in 10A.B2Cas cells than in 10A.B2Mock cells (Figure 3A). Moreover, to evaluate whether the enhanced activation of p70S6K is relevant for ErbB2-dependent invasiveness of 10A.B2Cas acini, we used the pharmacological inhibitor rapamycin, which specifically blocks p70S6K by acting on its upstream activator mTOR. Treatment with 20 nanomolar rapamycin was indeed sufficient to strongly reduce the phosphorylation of p70S6K on T389 and of its substrate S6Rp (Figure 3B) and to severely impair the formation of invasive protrusions in AP1510-treated 
10A.B2Cas multiacinar structures (Figure 3C), indicating that p70S6K plays a critical role in driving ErbB2-induced invasion in p130Cas over-expressing cells.

\section{p130Cas over-expression enhances ErbB2-dependent mTOR/p70S6K activation via Erk1/2} MAPK.

To understand the signalling pathways coordinated by p130Cas and ErbB2 resulting in activation of mTOR/p70S6K and leading to invasion, we examined the contribution of PI3K/Akt and Erk1/2 MAPK on p70S6K activation by using specific inhibitors. Specifically, to explore the effect of PI3K/Akt on p70S6K activity, 10A.B2Cas cells, left unstimulated or stimulated with AP1510, were treated with 10 micromolar LY294002. As shown in Figure 4, the pharmacological inhibition of $\mathrm{PI} 3 \mathrm{~K}$ results in the reduced phosphorylation of Akt on $\mathrm{S} 473$ and does not affect Erk1/2 phosphorylation. In parallel, p70S6K1 and its target S6Rp are still phosphorylated on T389 and S235/236, respectively, indicating that PI3K inhibition does not significantly alter p70S6K1 activation. Instead, the treatment with 25 micromolar of the MEK inhibitor PD98059 reduces Erk1/2 MAPK phosphorylation and impairs p70S6K and S6Rp phosphorylation, without affecting Akt phosphorylation. Taken together these data indicate that in response to ErbB2 activation, p130Cas over-expression enhances the mTOR/p70S6K pathway via Erk1/2 MAPK activation in a PI3K/Akt independent manner.

\section{p130Cas-dependent invasion of ErB2 transformed cells requires Rac1 activation via PI3K.}

It is known that p130Cas promotes actin remodelling by a conserved pathway involving the activation of the small GTPase Rac1 (Defilippi et al., 2006; Tikhmyanova et al., 2009). Notably, in this work, Rac1 activity was measured in $3 \mathrm{D}$ culture conditions by pull down assay from extracts of AP1510-stimulated 10A.B2Mock or 10A.B2Cas acini. The concomitant over-expression of p130Cas and ErbB2 activation lead to enhanced Rac1 activity compared to 10A.B2Mock (Figure 5A). Interestingly, inhibition of PI3K with LY294002 prevents the increase in Rac1 activity in 
response to AP1510 in 10A.B2Cas acini, indicating that p130Cas over-expression coupled to ErbB2 activation enhances Rac1 activity through the PI3K/Akt pathway (Figure 5A).

Finally, we examined the involvement of Rac1 in 3D ErbB2-mediated invasion of 10A.B2Cas acini by using 80 micromolar NSC23766, a specific Rac1 inhibitor. As shown in Figure 5B, the inhibition of Rac1 in AP1510-stimulated 10A.B2Cas abolished the formation of invasive protrusions into the surrounding matrix. Importantly, the inhibition of Rac1 does not affect upstream pathways as demonstrated by the phosphorylation of Akt, Erk1/2 or S6Rp (Figure 5C). In conclusion, our results demonstrate that in ErbB2 transformed 10A.B2Cas cells, the increased activity of Rac1 is dependent on PI3K activity and is required for the invasive behaviour of these cells.

Invasive p130Cas over-expressing 10A.B2 cells with activated ErbB2 form 3D matrix adhesions.

The interactions of cells with extracellular matrix (ECM) mediated by integrin receptors are $\underline{\text { required for many cellular processes including cell migration and invasion (Desgrosellier et }}$ al., 2010). As previously reported by Cukierman et al., 2001, fibroblasts grown in 3D are characterized by beta-1 integrin- and fibronectin-based adhesive structures defined as "3Dmatrix-adhesions" that totally differ in composition and function from 2D adhesive structures. To investigate the type of adhesive structures of 10A.B2Cas epithelial cells that, upon ErbB2 activation, escape from the acinar structures and invade the 3D matrix, we performed immunofluorescence experiments. 10A.B2Cas cells grown in 3D and stimulated with AP1510, were fixed and stained for beta-1 integrin, F-actin and fibronectin. As shown in Figure 6, at the invasive edge of the acini, cells form elongated adhesion fibrillar structures characterized by a strong co-localization between beta-1 integrin and fibronectin and intracellular bundles of F-actin parallel to extracellular fibronectin fibers. This is the first evidence that invading epithelial cells grown in three-dimensional cultures develop specific 3D 
matrix adhesions, that are different from the adhesive sites observed in 2D (data not shown), further underlining the relevance of 3D environment in regulating cell invasion dynamics. 


\section{Discussion}

In the present work we dissect the signalling pathways through which p130Cas confers invasive properties to ErbB-2 activated MCF10A.B2 cells by using a 3D model rather than the traditional monolayer culture. We show that the invasive behaviour of p130Cas over-expressing acini upon ErbB2 activation relies on the strengthening of Erk1/2 MAPK and PI3K/Akt activation. Interestingly, the treatment with pharmacological inhibitors indicates that these signalling pathways are both required in order to promote invasion of acinar structures. Moreover, we demonstrate that Erk1/2 MAPK and PI3K/Akt signalling confers invasive properties through activation of mTOR/ p70S6K and Rac1, as two distinct downstream effectors (Figure 7). Notably, invading epithelial cells over-expressing p130Cas and with activated ErbB2 are characterized by 3D matrix adhesions that have a distinct architecture compared to 2D adhesions.

MCF10A.B2 cells, that stably express a chimeric form of ErbB2, give rise to 3D multiacinar structures upon ErbB2 activation with a synthetic ligand. These multiacinar acini do not progress into an invasive phenotype (Muthuswamy et al., 2001). We showed that ErbB2-activated MCF10A.B2 cells acquire the ability to invade in 3D as the result of p130Cas over-expression, indicating that high levels of p130Cas are sufficient to drive invasiveness of ErbB2-positive mammary epithelial cells (Cabodi et al., 2010). However, the molecular events responsible for p130Cas-dependent invasiveness of ErbB2 transformed cells are not fully defined.

In monolayer cell cultures, the activation of ErbB2 receptors has been reported to induce signalling events, including sustained activation of c-Src, Erk1/2 MAPK and PI3K/Akt pathways that have been mainly related to cancer cell growth and survival (Graus-Porta et al., 1995; Hu et al., 1992; Karunagaran et al., 1996; Marcotte et al., 2009) as well as to cancer cell invasion (Ignatoski et al., 2000; Spencer et al., 2000). The intriguing and novel finding of the present work is that in a three dimensional model of mammary epithelial cells that upon ErbB2 activation closely mimics the in vivo mammary gland transformation, p130Cas over-expression strongly enhances Erk1/2 MAPK, PI3K/Akt, mTOR/p70S6K and Rac1 activation compared to control cells. All these signalling 
pathways are involved in mediating invasion of the mammary acini as demonstrated by the loss of invasive protrusions after the treatment with pharmacological inhibitors selective for each signalling pathway. It is worth noting that p130Cas over-expression by itself is not sufficient to enhance the levels of activation of Erk1/2 MAPK, PI3K/Akt, mTOR/p70S6K and Rac1 and to drive invasion, that indeed occurs only in presence of the additive activation of ErbB2. This suggests that the magnitude and stability of the signalling output in presence of p130Cas is not above the threshold necessary to initiate cell invasion, that is reached only in presence of ErbB2 stimulus. Interestingly, phosphorylation of c-Src, the major kinase known to phosphorylate p130Cas and a crucial transducer in ErbB2 signalling, is not enhanced by p130Cas over-expression in ErbB2 $\underline{\text { transformed acini (Figure S1B). In addition, c-Src kinase activity appears to be dispensable }}$ for driving invasion in 3D, as the treatment of mammary acini with the c-Src inhibitor SU6656 does not block cell invasion (Figure S1A). Consistently, Akt and Erk1/2 activities were not affected by the treatment with SU6656 (Figure S1B). Therefore, we can speculate that ErbB2-dependent c-Src activation is crucial for mediating transformation (Marcotte et al., 2009, Cabodi et a., 2010) but dispensable for eliciting cell invasion in 3D.

In this work, we identify the mTOR/p70S6K pathway, as a novel effector signalling of p130Casdependent invasion of ErbB2 transformed cells. Although it has been reported that both Erk1/2 MAPK and PI3K/Akt signalling pathways can lead to mTOR/p70S6K activation (Inoki et al., 2002; Kelleher et al., 2004; Lehman and Gomez-Cambronero, 2002; Sekulic et al., 2000), our results show that in a three-dimensional model the preferential pathway that induce mTOR/p70S6K activation depends on ErbB2/p130Cas/Erk1/2 MAPK cascade. This is supported by the fact that the treatment with MEK inhibitor markedly reduces p70S6K activation. In contrast, PI3K inhibition does not significantly affect Erk1/2 MAPK phosphorylation or p70S6K activation. Thus, p130Cas over-expression might preferentially conveys ErbB2 signalling to p70S6K through enhanced Erk1/2 MAPK activity that overcomes the PI3K/Akt pathway in the regulation of mTOR/p70S6K cascade. The fact that ErbB2 dependent activation of Akt in presence of over-expression of p130Cas is not 
involved in mTOR/p70S6K phosphorylation requires further investigation. Therefore, p130Cas over-expression further enhances the ErbB2-dependent activation of Erk1/2 MAPK thus promoting phosphorylation and activation of its downstream target mTOR/p70s6K that is required for cell invasion. Indeed, treatment with rapamycin, a selective inhibitor of mTOR, is sufficient to inhibit cell invasion.

Notably, it has been assessed that in human breast cancer, ErbB2 over-expression significantly correlates with p70S6K phosphorylation and this correlation was reported to be crucial for inducing angiogenesis and metastasis formation, underlining the clinical relevance of the activation of the mTOR/p70S6K in ErbB2 positive breast carcinoma (Klos et al., 2006).

In the metastatic process of cancer invasion, the degradation of the extracellular matrix is one of the most critical step (Deryugina and Quigley, 2006; Yu et al., 1997). It has been reported that p70S6K promotes cell migration and invasion of an ovarian cancer cell line over-expressing ErbB2 by upregulating transcription of Matrix Metalloproteinase-9 (MMP-9) (Zhou and Wong, 2006). Interestingly, MMP-9 activity is higher upon concomitant activation of ErbB2 and p130Cas overexpression in mammary epithelial cells grown as a monolayer culture (Cabodi et al., 2010). It is likely that one of the final targets of the mTOR/p70S6K signalling cascade may be the induction of MMP-9 that enables p130Cas over-expressing acini to invade into the extracellular matrix upon ErbB2 activation (Figure 7). Consistently, treatment with MMP-9 inhibitor, abrogates the formation of invasive protrusions in ErbB2 transformed acinar structures over-expressing p130Cas (data not shown).

In addition, our results identify Rac1 as a downstream effector of the PI3K/Akt pathway that is required for invasion of mammary epithelial acini. We have demonstrated that the concomitant over-expression of p130Cas and activation of ErbB2 drive Rac1 activity in 3D conditions. Interestingly, treatment with the PI3K inhibitor was sufficient to decrease Rac1 activation to control levels. Since we have shown that the PI3K inhibition does not affect the Erk1/2 MAPK pathway, it is likely that Erk1/2 MAPK are not involved in the enhanced activity of Rac1. In addition, we show 
that Rac1 is required for invasion, as demonstrated by the absence of invasive protrusions in response of Rac1 inhibitor. It is well documented that p130Cas plays a crucial role in regulating actin cytoskeleton remodelling by controlling Rac1 activity (Defilippi et al., 2006; Grimsley et al., 2004; Hsia et al., 2003). In addition, it has been reported that p130Cas can associate with the p85 subunit of PI3K in transformed cells (Li et al., 2000; Riggins et al., 2003). Therefore, we can speculate that in ErbB2 transformed mammary epithelial cells, high levels of p130Cas may facilitate the recruitment and activation of PI3K by ErbB2, that subsequently promote Rac1 activation, actin cytoskeleton re-organization, cell motility and invasion (Figure 7). Although it is known that the PI3K/Akt/Vav2 pathway can lead to the activation of Rac1 (King et al., 2000; Papakonstanti and Stournaras, 2002; Welch et al., 2003), the mechanisms that drive PI3Kdependent Rac1 activation in p130Cas over-expressing acini upon ErbB2 activation remain to be elucidated.

Overall, it is important to highlight that neither the Erk1/2 MAPK/p70S6K nor the PI3K/Akt/Rac1 pathway is sufficient by itself to elicit cell invasion. Indeed, the inhibition of either one pathway blocks the invasive process without affecting the other one. Therefore, the invasive process may be the result of the activation of Erk1/2 MAPK/p70S6K and PI3K/Akt/Rac1 pathways that target not overlapping effectors involved in distinct functional events. Alternatively, these two signalling pathways may converge on the same downstream effector, whose activation occurs only in presence of the additive effects of both signalling pathways.

Importantly, the results of the in silico analysis that we performed by using publicly available microarray data from the Netherlands Cancer Institute, (van de Vijver et al., 2002) indicate that patients with ErbB2 positive breast cancers and expressing high levels of p130Cas have a higher risk of developing metastasis and a shorter overall survival compared to patients with ErbB2 positive breast cancers for but with lower levels of p130Cas expression. These data suggest that expression of p130Cas might serve as indicator of ErbB2 breast cancer progression. 
In summary, our data emphasize the crucial role of the concomitant over-expression of p130Cas and ErbB2 activation in promoting cell invasion. Our data suggest that high levels of p130Cas expression function as a molecular switch that by amplifying signals from active ErbB2 and intracellular cues promotes the progression from the proliferative to the invasive behaviour, pointing out a possible role for p130Cas as a predictive marker for ErbB2 breast cancer progression. Most importantly, as p130Cas controls several ErbB2 activated signalling pathways, its targeting is likely to be effective for the treatment of ErbB2 over-expressing and invasive breast tumours.

\section{Acknowledgments}

We thank Dr. S. Muthuswamy for 10A.B2 cells and Agata Tinnirello for technical advises. We thank Emanuela Santopietro and Elisa De Luca for technical help with the confocal microscopy. M.P Camacho-Leal is supported by the Association for International Cancer research (AICR). This work was supported by Italian Association for Cancer Research (AIRC), AICR, EU FP7 Metafight, MUR (Ministero deIl'Università e Ricerca Scientifica, PRIN, ex-60\%), Regione Piemonte Progetti Sanità, Oncoprot, Pi STEM, Druidi and CIPE, Compagnia San Paolo, Progetto AlfieriFondazione CRT, Torino. 


\section{References}

Allgayer, H., Babic, R., Gruetzner, K.U., Tarabichi, A., Schildberg, F.W., Heiss, M.M., 2000. cerbB-2 is of independent prognostic relevance in gastric cancer and is associated with the expression of tumor-associated protease systems. J Clin Oncol 18, 2201-2209.

Baselga, J., Swain, S.M., 2009. Novel anticancer targets: revisiting ERBB2 and discovering ERBB3. Nat Rev Cancer 9, 463-475.

Bouton, A.H., Riggins, R.B., Bruce-Staskal, P.J., 2001. Functions of the adapter protein Cas: signal convergence and the determination of cellular responses. Oncogene 20, 6448-6458.

Cabodi, S., Tinnirello, A., Di Stefano, P., Bisaro, B., Ambrosino, E., Castellano, I., Sapino, A., Arisio, R., Cavallo, F., Forni, G., Glukhova, M., Silengo, L., Altruda, F., Turco, E., Tarone, G., Defilippi, P., 2006. p130Cas as a new regulator of mammary epithelial cell proliferation, survival, and HER2-neu oncogene-dependent breast tumorigenesis. Cancer Res 66, 4672-4680.

Cabodi, S., Tinnirello, A., Bisaro, B., Tornillo, G., Camacho-Leal M.D.P., Forni, G., Cojoca, R., Iezzi, M., Amici, A., Montani, M., Eva, A., Di Stefano, P., Muthuswamy, S.K., Tarone, G., Turco, E., Defilippi, P., 2010. p130Cas is an essential transducer element in ErbB2 transformation. FASEB J. [Epub ahead of print]

Cukierman, E., Pankov, R., Stevens, D.R., Yamada, K.M., 2001. Taking cell-matrix adhesions to the third dimension. Science. 294:1708-1712.

Debnath, J., Muthuswamy, S.K., Brugge, J.S., 2003. Morphogenesis and oncogenesis of MCF-10A mammary epithelial acini grown in three-dimensional basement membrane cultures. Methods 30, 256-268.

Defilippi, P., Di Stefano, P., Cabodi, S., 2006. p130Cas: a versatile scaffold in signaling networks. Trends Cell Biol 16, 257-263.

Deryugina, E.I., Quigley, J.P., 2006. Matrix metalloproteinases and tumor metastasis. Cancer Metastasis Rev 25, 9-34. 
Desgrosellier, J.S., Cheresh, D.A., 2010. Integrins in cancer: biological implications and therapeutic opportunities. Nat Rev Cancer 10, 9-22.

Di Stefano, P., Damiano, L., Cabodi, S., Aramu, S., Tordella, L., Praduroux, A., Piva, R., Cavallo, F., Forni, G., Silengo, L., Tarone, G., Turco, E., Defilippi, P., 2007. p140Cap protein suppresses tumour cell properties, regulating Csk and Src kinase activity. EMBO J 26, 2843-2855.

Graus-Porta, D., Beerli, R.R., Hynes, N.E., 1995. Single-chain antibody-mediated intracellular retention of ErbB-2 impairs Neu differentiation factor and epidermal growth factor signaling. Mol Cell Biol 15, 1182-1191.

Grimsley, C.M., Kinchen, J.M., Tosello-Trampont, A.C., Brugnera, E., Haney, L.B., Lu, M., Chen, Q., Klingele, D., Hengartner, M.O., Ravichandran, K.S., 2004. Dock180 and ELMO1 proteins cooperate to promote evolutionarily conserved Rac-dependent cell migration. J Biol Chem 279, 6087-6097.

Hay, N., Sonenberg, N., 2004. Upstream and downstream of mTOR. Genes Dev 18, 1926-1945.

Holland, E.C., Sonenberg, N., Pandolfi, P.P., Thomas, G., 2004. Signaling control of mRNA translation in cancer pathogenesis. Oncogene 23, 3138-3144.

Honda, H., Oda, H., Nakamoto, T., Honda, Z., Sakai, R., Suzuki, T., Saito, T., Nakamura, K., Nakao, K., Ishikawa, T., Katsuki, M., Yazaki, Y., Hirai, H., 1998. Cardiovascular anomaly, impaired actin bundling and resistance to Src-induced transformation in mice lacking p130Cas. Nat Genet 19, 361-365.

Hsia, D.A., Mitra, S.K., Hauck, C.R., Streblow, D.N., Nelson, J.A., Ilic, D., Huang, S., Li, E., Nemerow, G.R., Leng, J., Spencer, K.S., Cheresh, D.A., Schlaepfer, D.D., 2003. Differential regulation of cell motility and invasion by FAK. J Cell Biol 160, 753-767.

Hu, P., Margolis, B., Skolnik, E.Y., Lammers, R., Ullrich, A., Schlessinger, J., 1992. Interaction of phosphatidylinositol 3-kinase-associated p85 with epidermal growth factor and platelet-derived growth factor receptors. Mol Cell Biol 12, 981-990. 
Hynes, N.E., Lane, H.A., 2005. ERBB receptors and cancer: the complexity of targeted inhibitors. Nat Rev Cancer 5, 341-354.

Hynes, N.E., MacDonald, G., 2009. ErbB receptors and signaling pathways in cancer. Curr Opin Cell Biol 21, 177-184.

Ignatoski, K.M., Maehama, T., Markwart, S.M., Dixon, J.E., Livant, D.L., Ethier, S.P., 2000. ERBB-2 overexpression confers PI 3' kinase-dependent invasion capacity on human mammary epithelial cells. Br J Cancer 82, 666-674.

Inoki, K., Li, Y., Zhu, T., Wu, J., Guan, K.L., 2002. TSC2 is phosphorylated and inhibited by Akt and suppresses mTOR signalling. Nat Cell Biol 4, 648-657.

Karunagaran, D., Tzahar, E., Beerli, R.R., Chen, X., Graus-Porta, D., Ratzkin, B.J., Seger, R., Hynes, N.E., Yarden, Y., 1996. ErbB-2 is a common auxiliary subunit of NDF and EGF receptors: implications for breast cancer. EMBO J 15, 254-264.

Kelleher, R.J., 3rd, Govindarajan, A., Jung, H.Y., Kang, H., Tonegawa, S., 2004. Translational control by MAPK signaling in long-term synaptic plasticity and memory. Cell 116, 467-479.

King, C.C., Gardiner, E.M., Zenke, F.T., Bohl, B.P., Newton, A.C., Hemmings, B.A., Bokoch, G.M., 2000. p21-activated kinase (PAK1) is phosphorylated and activated by 3-phosphoinositidedependent kinase-1 (PDK1). J Biol Chem 275, 41201-41209.

Klemke, R.L., Leng, J., Molander, R., Brooks, P.C., Vuori, K., Cheresh, D.A., 1998. CAS/Crk coupling serves as a "molecular switch" for induction of cell migration. J Cell Biol 140, 961-972.

Klos, K.S., Wyszomierski, S.L., Sun, M., Tan, M., Zhou, X., Li, P., Yang, W., Yin, G., Hittelman, W.N., Yu, D., 2006. ErbB2 increases vascular endothelial growth factor protein synthesis via activation of mammalian target of rapamycin/p70S6K leading to increased angiogenesis and spontaneous metastasis of human breast cancer cells. Cancer Res 66, 2028-2037.

Laplante, M., Sabatini, D.M., 2009. mTOR signaling at a glance. J Cell Sci 122, 3589-3594.

Lehman, J.A., Gomez-Cambronero, J., 2002. Molecular crosstalk between p70S6k and MAPK cell signaling pathways. Biochem Biophys Res Commun 293, 463-469. 
Li, E., Stupack, D.G., Brown, S.L., Klemke, R., Schlaepfer, D.D., Nemerow, G.R., 2000. Association of p130CAS with phosphatidylinositol-3-OH kinase mediates adenovirus cell entry. J Biol Chem 275, 14729-14735.

Ma, X.M., Blenis, J., 2009. Molecular mechanisms of mTOR-mediated translational control. Nat Rev Mol Cell Biol 5, 307-318.

Marcotte, R., Zhou, L., Kim, H., Roskelly, C.D., Muller, W.J., 2009. c-Src associates with ErbB2 through an interaction between catalytic domains and confers enhanced transforming potential. Mol Cell Biol 29, 5858-5871.

Matsuda, M., Mayer, B.J., Fukui, Y., Hanafusa, H., 1990. Binding of transforming protein, P47gagcrk, to a broad range of phosphotyrosine-containing proteins. Science 248, 1537-1539.

Mitra, S.K., Schlaepfer, D.D., 2006. Integrin-regulated FAK-Src signaling in normal and cancer cells. Curr Opin Cell Biol 18, 516-523.

Muller, W.J., Sinn, E., Pattengale, P.K., Wallace, R., Leder, P., 1988. Single-step induction of mammary adenocarcinoma in transgenic mice bearing the activated c-neu oncogene. Cell 54, 105115.

Muthuswamy, S.K., Li, D., Lelievre, S., Bissell, M.J., Brugge, J.S., 2001. ErbB2, but not ErbB1, reinitiates proliferation and induces luminal repopulation in epithelial acini. Nat Cell Biol 3, 785792.

Nanni, P., Pupa, S.M., Nicoletti, G., De Giovanni, C., Landuzzi, L., Rossi, I., Astolfi, A., Ricci, C., De Vecchi, R., Invernizzi, A.M., Di Carlo, E., Musiani, P., Forni, G., Menard, S., Lollini, P.L., 2000. p185(neu) protein is required for tumor and anchorage-independent growth, not for cell proliferation of transgenic mammary carcinoma. Int J Cancer 87, 186-194.

Neve, R.M., Holbro, T., Hynes, N.E., 2002. Distinct roles for phosphoinositide 3 -kinase, mitogenactivated protein kinase and p38 MAPK in mediating cell cycle progression of breast cancer cells. Oncogene 21, 4567-4576. 
O'Neill, G.M., Fashena, S.J., Golemis, E.A., 2000. Integrin signalling: a new Cas(t) of characters enters the stage. Trends Cell Biol 10, 111-119.

Papakonstanti, E.A., Stournaras, C., 2002. Association of PI-3 kinase with PAK1 leads to actin phosphorylation and cytoskeletal reorganization. Mol Biol Cell 13, 2946-2962.

Pearson, R.B., Dennis, P.B., Han, J.W., Williamson, N.A., Kozma, S.C., Wettenhall, R.E., Thomas, G., 1995. The principal target of rapamycin-induced p70s6k inactivation is a novel phosphorylation site within a conserved hydrophobic domain. EMBO J 14, 5279-5287.

Reynolds, A.B., Kanner, S.B., Wang, H.C., Parsons, J.T., 1989. Stable association of activated pp60src with two tyrosine-phosphorylated cellular proteins. Mol Cell Biol 9, 3951-3958.

Riggins, R.B., Quilliam, L.A., Bouton, A.H., 2003. Synergistic promotion of c-Src activation and cell migration by Cas and AND-34/BCAR3. J Biol Chem 278, 28264-28273.

Saitoh, M., Pullen, N., Brennan, P., Cantrell, D., Dennis, P.B., Thomas, G., 2002. Regulation of an activated S6 kinase 1 variant reveals a novel mammalian target of rapamycin phosphorylation site. J Biol Chem 277, 20104-20112.

Sekulic, A., Hudson, C.C., Homme, J.L., Yin, P., Otterness, D.M., Karnitz, L.M., Abraham, R.T., 2000. A direct linkage between the phosphoinositide 3-kinase-AKT signaling pathway and the mammalian target of rapamycin in mitogen-stimulated and transformed cells. Cancer Res 60, 35043513.

Sharma, S.V., Settleman, J., 2009. ErbBs in lung cancer. Exp Cell Res 315, 557-571.

Slamon, D.J., Godolphin, W., Jones, L.A., Holt, J.A., Wong, S.G., Keith, D.E., Levin, W.J., Stuart, S.G., Udove, J., Ullrich, A., et al., 1989. Studies of the HER-2/neu proto-oncogene in human breast and ovarian cancer. Science 244, 707-712.

Spencer, K.S., Graus-Porta, D., Leng, J., Hynes, N.E., Klemke, R.L., 2000. ErbB2 is necessary for induction of carcinoma cell invasion by ErbB family receptor tyrosine kinases. J Cell Biol 148, 385397. 
Stupack, D.G., Cho, S.Y., Klemke, R.L., 2000. Molecular signaling mechanisms of cell migration and invasion. Immunol Res 21, 83-88.

Ta, H.Q., Thomas, K.S., Schrecengost, R.S., Bouton, A.H., 2008. A novel association between p130Cas and resistance to the chemotherapeutic drug adriamycin in human breast cancer cells. Cancer Res 68, 8796-8804.

Thomas, G., 1993. p70s6k/p85s6k: mechanism of activation, effects of rapamycin and role in mitogenesis. Biochem Soc Trans 21, 901-904.

Tikhmyanova, N., Little, J.L., Golemis, E.A., 2009. CAS proteins in normal and pathological cell growth control. Cell Mol Life Sci.

van de Vijver, M.J., He, Y.D., van't Veer, L.J., Dai, H., Hart, A.A., Voskuil, D.W., Schreiber, G.J., Peterse, J.L., Roberts, C., Marton, M.J., Parrish, M., Atsma, D., Witteveen, A., Glas, A., Delahaye, L., van der Velde, T., Bartelink, H., Rodenhuis, S., Rutgers, E.T., Friend, S.H., Bernards, R., 2002. A gene-expression signature as a predictor of survival in breast cancer. N Engl J Med 347, 19992009.

van der Flier, S., Brinkman, A., Look, M.P., Kok, E.M., Meijer-van Gelder, M.E., Klijn, J.G., Dorssers, L.C., Foekens, J.A., 2000. Bcar1/p130Cas protein and primary breast cancer: prognosis and response to tamoxifen treatment. J Natl Cancer Inst 92, 120-127.

Welch, H.C., Coadwell, W.J., Stephens, L.R., Hawkins, P.T., 2003. Phosphoinositide 3-kinasedependent activation of Rac. FEBS Lett 546, 93-97.

Yarden, Y., Sliwkowski, M.X., 2001. Untangling the ErbB signalling network. Nat Rev Mol Cell Biol 2, 127-137.

Yu, A.E., Hewitt, R.E., Connor, E.W., Stetler-Stevenson, W.G., 1997. Matrix metalloproteinases. Novel targets for directed cancer therapy. Drugs Aging 11, 229-244.

Zhou, H.Y., Wong, A.S., 2006. Activation of p70S6K induces expression of matrix metalloproteinase 9 associated with hepatocyte growth factor-mediated invasion in human ovarian cancer cells. Endocrinology 147, 2557-2566. 


\section{Figure legends}

\section{Figure 1: In silico analysis of ErbB2 positive human breast cancer}

A) Higher levels of expression of ErbB2 are detected in patients with high levels of p130Cas $\left(\mathrm{p} 130 \mathrm{Cas} / \mathrm{BCAR}^{+}{ }^{+}\right.$compared to patients with low levels of p130Cas (p130Cas/BCAR1') (**p $<0.001$ from Mann-Whithney $\mathrm{U}$ test) as shown in the boxplot.

(B and C) Patients with both high levels of ErbB2 and p130Cas had a significant reduced survival $(B ; p=0.012)$ and increased risk of developing metastasis $(C ; p=0.045)$ compared to patients with both low levels of ErbB2 and p130Cas as shown by Kaplan-Meier curves.

Figure 2: Erk1/2 MAPK and PI3K/Akt pathways are required for p130Cas and ErbB2dependent invasion of MCF10A.B2 cells grown in 3D.

(A) 10A.B2Mock and 10A.B2Cas cells were plated on a matrix generated by mixing Matrigel and collagen I in a 1:1 ratio and stimulated with vehicle or 1 micromolar AP1510 on day 12, 15 and 18. On day 19, acinar structures were recovered and lysed. Total cell extracts were analyzed by immunoblot and probed with antibodies to p130Cas, pAkt S473, Akt, pErk1/2 and Erk1/2. Right: Quantification analysis of three independent experiments. Statistical analysis was performed by Student's $t$ test $(* \mathrm{p}<0.05, * * \mathrm{p}<0.01)$.

(B) Representative phase images of day-19 acinar structures formed by 10A.B2Mock (upper panels), 10A.B2Cas (middle panels) cells un-stimulated or stimulated with AP1510 and 10A.B2Cas cells stimulated with AP1510 and treated with PD98059 (25 micromolar) or LY294002 (10 micromolar) (lower panels). Scale bars, $100 \mu \mathrm{m}$.

Figure 3: p130Cas/ErbB2 dependent invasion requires mTOR/p70S6K activity.

(A) 10A.B2Mock and 10A.B2Cas cells were cultured as described in Fig. 2A. Total cell extracts were analyzed by immunoblot and probed with antibodies to phospho-p70S6K (pS6K T421/S424 and T389), p70S6K (S6K), phospho-S6Rp (pS6Rp S235/236) and S6Rp. Right: Quantification 
analysis of three independent experiments. Statistical analysis was performed by Student's $t$ test $\left({ }^{*} \mathrm{p}<0.05, * * \mathrm{p}<0.01\right)$.

(B) 10A.B2Cas acini were cultured as in (A) and stimulated with AP1510 (1 micromolar) in the presence of rapamycin (20 nanomolar). Total cell extracts were analyzed by immunoblot and probed with antibodies to phospho-p70S6K (pS6K T389), p70S6K (S6K), phospho-S6Rp (pS6Rp S235/236) and S6Rp.

(C) Representative phase images of day-19 acinar structures formed by un-stimulated 10A.B2Mock and 10A.B2Cas acinar structures (left panels), 10A.B2Mock and 10A.B2Cas stimulated acini with AP1510 (middle panels) and 10A.B2Cas cells stimulated with AP1510 and treated with rapamycin (20 nanomolar) (right panel). Scale bars, $100 \mu \mathrm{m}$.

Figure 4: p130Cas over-expression enhances ErbB2-dependent mTor activation via Erk1/2 MAPK.

(A) Western blot analysis was performed on cell extracts from un-stimulated or AP1510-stimulated 10A.B2Mock and 10A.B2Cas acini and from 10A.B2Cas acini stimulated with AP1510 and treated with PD98059 (25 micromolar) or LY294002 (10 micromolar). Cell extracts were probed with antibodies to pAkt S473, Akt, pErk1/2, Erk1/2, phospho-p70S6K (pS6K T389), p70S6K (S6K), phospho-S6Rp (pS6Rp S235/236) and S6Rp.

(B) Quantification analysis of three independent experiments. Statistical analysis was performed by Student's $t$ test $(* p<0.05, * * p<0.01)$.

Figure 5: Rac1 inhibition is sufficient to abrogate invasion of ErbB2 transformed 10A.B2Cas acini.

(A) 10A.B2Mock and 10A.B2Cas acini were stimulated with AP1510 on day 12 and 15, in presence or absence of LY294002 (10 micromolar), and lysed on day 16. Active Rac1 was pulled down from total protein extracts using the CRIB domain of PAK and detected by Western blot with an anti-Rac1 mAb (upper panel). Amount of total Rac1 in protein extracts is shown in the lower 
panel. Right panel: Quantification analysis of three independent experiments. Statistical analysis was performed by Student's $t$ test $(* \mathrm{p}<0.05)$.

(B) Representative phase images of day-19 acinar structures formed by unstimulated 10A.B2Mock and 10A.B2Cas acinar structures (left panels), 10A.B2Mock and 10A.B2Cas stimulated acini with AP1510 (middle panels) and 10A.B2Cas cells stimulated with AP1510 and treated with NSC23766 (80 micromolar) (right panel). Scale bars, $100 \mu \mathrm{m}$.

(C) 10A.B2Mock and 10A.B2Cas cells were cultivated as described in Fig. 2A. 10A.B2Cas acini were stimulated with AP1510 in the presence of NSC23766 (80 micromolar). Total cell extracts were probed with antibodies to pAkt S473, Akt, pErk1/2, Erk1/2, phospho-S6Rp (pS6Rp S235/236) and S6Rp.

Figure 6: ErbB2 transfomed 10A.B2Cas invasive cells are characterized by "3D matrix adhesions".

Representative confocal images of AP1510-stimulated 10A.B2Cas acini fixed and stained for F-actin (purple, panel a), beta-1 integrin (red, panel b) and fibronectin (green, panel c). Merged images are shown in panel d. Magnifications of the insets are shown in panels $e, f, g$ and $h$, respectively. White arrows in panel $h$ indicate co-localization between beta-1 integrin and fibronectin. Scale bars represent $25 \mu \mathrm{m}$. Images were taken at HCX PL APO CS 63X 1.4 OIL Leica TCS-SP5 II confocal microscope and analyzed with LASAF software.

Figure 7: Signaling pathways leading to 3D invasion of ErbB2 transformed 10A.B2Cas acini. In ErbB2 transformed 10A.B2 mammary epithelial cells, the over-expression of p130Cas triggers invasion by acting on both the PI3K/Akt and Erk1/2 pathways. mTOR/p70S6K signalling pathways is preferentially activated by the ErbB2/p130Cas/Erk1/2 MAPK cascade. Treatment with MEK inhibitor abrogates cell invasion by affecting mTOR/p70S6K pathway. On the other side, PI3K inhibition blocks cell invasion but does not significantly affect Erk1/2 MAPK phosphorylation or p70S6K activation. In parallel, the ErbB2/p130Cas/PI3K/Akt cascade triggers Rac1 activation. Both signalling pathways are required for epithelial cell 
invasion suggesting that they cooperate in the regulation of different processes that ultimately

lead to cell invasion. 